\title{
Bi-modal distribution of stabilizers to regulate the dual-phase microstructure and transformability in $\mathrm{Nd}_{2} \mathrm{O}_{3} / \mathrm{Y}_{2} \mathrm{O}_{3}$-doped \\ zirconia ceramics
}

\author{
Wei Chen ${ }^{1}$, Dong-Li Hu${ }^{1 *}$, Hui Gu ${ }^{1 *}$, Peng-Xiang Qian ${ }^{2 \#}$, Jef Vleugels ${ }^{3}$, \\ Ying Jiang ${ }^{1}$, Juan-Juan Xing ${ }^{1}$ \\ ${ }^{1}$ Materials Genome Institute, School of Materials Science and Engineering, Shanghai \\ University, Shanghai 200444, China \\ 2 State Key Laboratory of High Performance Ceramics and Superfine Microstructures, \\ Shanghai Institute of Ceramics, Chinese Academy of Sciences, Shanghai 200050, \\ China \\ ${ }^{3}$ KU Leuven, Department of Materials Engineering, Arenberg Kasteelpark 44, B- \\ 3001, Heverlee, Belgium
}

\begin{abstract}
The phase relationship and microstructural characteristics were systematically studied for co-stabilized $\mathrm{ZrO}_{2}$ ceramics with $\mathrm{Nd}_{2} \mathrm{O}_{3}-\mathrm{Y}_{2} \mathrm{O}_{3}$ additives coated onto starting powder. Polishing induced surface morphological changes by tetragonal-to-monoclinic transformation were observed by SEM. EDS analysis revealed a bi-modal distribution of the stabilizers correspondingly to smaller transformable tetragonal grains and larger non-transformable cubic grains. The correlation of co-stabilizer concentrations with the dual-phase microstructures leads to a probable two-stage sintering enabled by transient liquid-phase started from coating. Small $t-\mathrm{ZrO}_{2}$ grains inherited the starting nanopowder to take less stabilizers via diffusion, and larger $c-\mathrm{ZrO}_{2}$ grains incorporate directly stabilizers during growth from liquid-phase accumulated more stabilizers. The transformability of $t-\mathrm{ZrO}_{2}$ grains is influenced by neighboring $c-\mathrm{ZrO}_{2}$ grains when a compressive stress on the former is sufficiently large from the latter in larger size and high modulus, which could be regulated by the two-stage process to optimize the dual-
\end{abstract}

\footnotetext{
* Corresponding author. E-mail address: dlhu_sh@t.shu.edu.cn (D.L. Hu).

* Corresponding author. E-mail address: gujiaoshou@t.shu.edu.cn (H. Gu).

\# Present address: SCHOTT Glass Technologies (Suzhou) Co., Ltd., Suzhou, China
} 
phase microstructure.

Keywords: Dual-phase microstructure; Bi-modal distribution of stabilizers; Transformability; Transient liquid-phase; Surface treatment

\section{Introduction}

Zirconia ceramics with tetragonal phase (TZP) have excellent flexural strength and fracture toughness owing to a stress-induced tetragonal-to-monoclinic $(t \rightarrow m)$ transformation upon fracture, hence they are used widely from body implants and dental applications to thermal-barrier-coatings (TBC) for several decades $[1,2]$. The transformable $t-\mathrm{ZrO}_{2}$ phase is stabilized by rare-earth dopants in Ce-TZP and especially Y-TZP, and the grain size is directly correlated with the $t$-phase transformability and the ceramic toughness $[3,4]$, which is also true in nano-crystalline Y-TZP [5]. Furthermore, an inhomogeneous distribution of the stabilizer doped via the powder coating route could effectively enhance the toughness [6]. Indeed, the stabilizer level was found to increase inversely with the grain size in Ce-TZP, which indicates that the sintering process could control not only the grain size but also the distribution of the stabilizer [7].

Most Y-TZP ceramics suffer from low-temperature degradation (LTD) when exposed to ambient humidity, which severely limits its application as biomaterial $[8,9]$. Addition of Ce as co-stabilizer could effectively eliminate the LTD effect in Ce/Y-TZP, and the powder coating approach retained superior mechanical properties $[10,11]$. In comparison, better mechanical properties and phase stability were demonstrated for $\mathrm{Nd} / \mathrm{Y}-\mathrm{TZP}$ ceramics presureless sintered at $1400-1450^{\circ} \mathrm{C}$ from stabilizer-coated starting powder at relatively low stabilizer levels [12-14]. The effect of the co-stabilizer distribution in the microstructure on the $t$-phase transformability and hence the fracture toughness and hydrothermal stability however is still unknown. In this work, we will study the phase relations in Nd/Y-TZP ceramics, especially focusing on the stabilizer 
distribution by micro-chemical analysis in a scanning (SEM) and transmission electron microscope (TEM). Dual-phase $(t / c)$ microstructure is found to dominate the costabilized TZP ceramics, which is similar to recent observation in Y-TZP but will be rationalized with rather different mechanism [15].

\section{Experimental}

$\mathrm{ZrO}_{2}$ powder was coated with 0.1 molar $\mathrm{Nd}\left(\mathrm{NO}_{3}\right)_{3} \cdot 6 \mathrm{H}_{2} \mathrm{O}(99.9 \%$, Aldrich) and $\mathrm{Y}\left(\mathrm{NO}_{3}\right)_{3} \cdot 6 \mathrm{H}_{2} \mathrm{O}(99.9 \%$, Aldrich) solutions. The appropriate amount of solution was mixed on a multidirectional mixer (Turbula type) with pure $m-\mathrm{ZrO}_{2}$ powder (Tosoh grade TZ-0, $27 \mathrm{~nm}$, Japan) in ethanol in a polyethylene container for $24 \mathrm{~h}$ with 3Y-TZP milling balls to break the agglomerates. After calcination at $800^{\circ} \mathrm{C}$ for 1 hour, another mixing was performed in ethanol to break agglomerates of stabilizer-coated $\mathrm{ZrO}_{2}$ nanopowder. After cold isostatic pressing at $300 \mathrm{MPa}$, the green bodies were sintered into fully densified ceramics in a tube furnace (TGA-171, Cahn, USA) for $2 \mathrm{~h}$ at $1450^{\circ} \mathrm{C}$ under an air flow of $100 \mathrm{ml} / \mathrm{min}$. Two Nd/Y-TZP ceramics were investigated in this study, both with 2 mol. $\% \mathrm{Nd}_{2} \mathrm{O}_{3}$ but with a different $\mathrm{Y}_{2} \mathrm{O}_{3}$ amount of 1.5 and $2.5 \mathrm{~mol} . \%$, referred to as 4302 and 4502 according to the corresponding $\mathrm{NdO}_{1.5}$ and $\mathrm{YO}_{1.5}$ mole contents and holding time of 2 hours at the sintering temperature. Further details about the material processing and physical properties are reported elsewhere [14].

Although the microstructures of various $\mathrm{Nd} / \mathrm{Y}-\mathrm{TZP}$ ceramics were briefly compared 15 years ago, we re-examined detailed microstructural features of these two samples by a combination of SEM (model G300, Carl Zeiss, Germany) and TEM (model JEM-2100F, JEOL, Japan) imaging and analytical capabilities. Besides secondary electron (SE) and backscattered electron (BSE) imaging modes, this SEM is also equipped with an energy dispersive X-ray spectrometer (model AZtec, Oxford Instrument, UK) and electron backscatter diffraction (EBSD, Oxford, UK) systems to 
analyze the local chemistry and crystal structures. EDS point analysis in SEM was performed with an accelerating voltage of electron probe under $10 \mathrm{kV}$ to include all cations into the quantitative analysis using standards from the commercial system. Elemental mapping was performed under $5 \mathrm{kV}$ to achieve better lateral resolution. EBSD mapping was performed under $20 \mathrm{kV}$ and a step size of $30 \mathrm{~nm}$ to guarantee sufficient signal intensity.

Bulk phase identification on mechanical polished surface was carried out using Xray diffraction (model D2, Bruker, Germany) with a scan-step of $0.02^{\circ}$, enabling to identify and quantify the different $\mathrm{ZrO}_{2}$ phases. The phase contents were calculated from the relative intensities of corresponding reflections by commercial software (JADE). The TEM was operated under an accelerating voltage of $200 \mathrm{kV}$, and equipped with an X-ray spectrometer (model EDAX, Edax Instrument, USA) for EDS analysis. Quantification of cation ratio in TEM was processed in a standard-less mode using the thin-specimen approach. TEM specimens were prepared by ion milling (model EM RES101, Leica, Germany) after cutting, polishing and dimpling. For better imaging and analysis, all samples were coated with a $\sim 3 \mathrm{~nm}$ thin carbon coating (model EM ACE600, Leica, Germany).

Most studies on TZP microstructures use thermal etching to highlight the grain morphology after mechanical polishing. In this study, the triple ion-beam cutting technology (model EM TIC 3X, Leica, Germany) was used to expose the native surface for imaging and EDS analysis to minimize the effect from mechanical polishing instead. This implies an ion slope cutting (ISC) performed under $6.5 \mathrm{kV}$ at $0^{\circ}$ tilt for $3.5 \mathrm{~h}$ for each sample surface. Vibration polishing (VP, model VibroMet 2, USA) was applied on the sample surface for EBSD analysis. In addition to the conventional TEM specimen preparation for both ceramic samples, a TEM cross-sectional specimen was thinned by focused ion-beam equipment (FIB, model Helios G4 UX, Thermo Fisher Scientific, China) from a vibration polished 4302 surface. 


\section{Results}

\subsection{Phase identification by SEM imaging}

The highest fracture toughness among a series of co-stabilizer contents and sintering times was measured for the 4302 ceramic, and the absence of $m-\mathrm{ZrO}_{2}$ phase implies that there was only transformable $t-\mathrm{ZrO}_{2}$ phase co-existing with $c-\mathrm{ZrO}_{2}$ phase [14]. As shown in Fig. 1 for both 4302 and 4502, ion cutting reveals the grain

morphology of the $\mathrm{ZrO}_{2}$ ceramics in both SE and BSE mode images, even though the SE mode images clearly indicate that the ISC surfaces are not as flat as other surfaces. Furthermore and especially in the BSE images, not only the grains could be further distinguished by a bright and dark contrast, but there are also gray contrast grains, especially in 4302 . These gray grains often exhibit a twinned structure either below or above the surface, and the latter appears as a striped pattern as marked by the red circles. This twin-structure can only be attributed to the presence of $m$-phase in the Nd/Y-TZP ceramics. The fact that some of these nanometric $m$-phase grains are protruding out of the surface is due to the volume expansion upon transformation from the initial $t$-phase grains. 

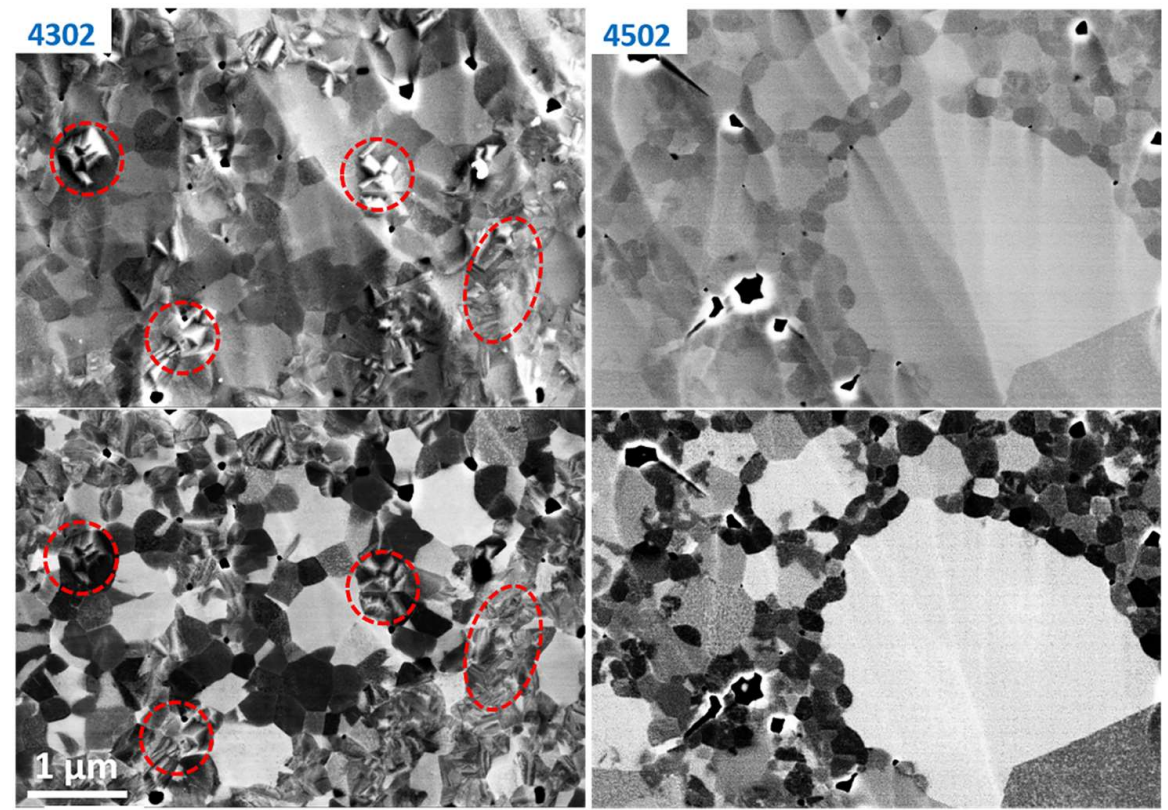

Fig. 1 SE/BSE (upper/lower panels) images of ion slope cut 4302 and 4502 Nd/Y-TZP ceramics revealing bright (large), dark (smaller) and gray contrast $\mathrm{ZrO}_{2}$ grains. The gray contrast grains in 4302 have a twinned appearance, as marked by the red circles, which is much less pronounced in 4502 .

EBSD analysis of a flat polished 4302 surface revealed that the bright and dark contrast phases correspond to $c$ - and $m$-phase respectively as shown in Fig. 2, while almost no $t$-phase was detected. The large grains correspond mainly to $c$-phase, and a small number of points inside these grains are recognized mistakenly as $t$-phase. Analysis of small grains fails at the majority of black points to manifest as zero resolution, and all the non-zero points corresponds to $m$-phase, regardless of small grain size and even smaller twin features that are hard to resolve by EBSD. The dark grains in 4502 are smaller hence even harder to know whether they are transformed or not, while the bright grains are all in $c$-phase. 


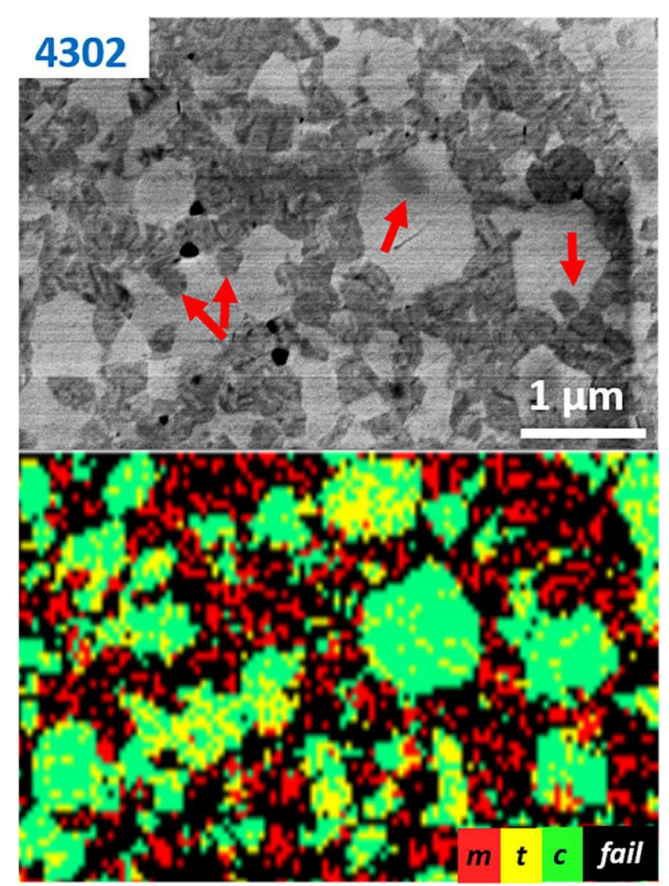

Fig. 2 BSE image (upper) and corresponding EBSD mapping (lower) for phase identification of the individual $\mathrm{ZrO}_{2}$ grains in the 4302 ceramic.

To assess whether some or all $t$-phase grains transformed into $m$-phase grains during 15 years staying in ambient condition in a desiccator to prevent excessive humidity, high-resolution XRD analysis on mechanically polished surfaces was performed on both ceramics. The $t$ - and $c-\mathrm{ZrO}_{2}$ remained as primary phases as shown in Fig. 3, even though weak $m-\mathrm{ZrO}_{2}$ peaks were also detected. Both $\mathrm{Nd} / \mathrm{Y}-\mathrm{TZP}$ ceramics are largely untransformed to signify a mild LTD effect, as compared to the XRD analysis of the as-sintered samples where no $m$-phase was detected [16-18]. Therefore, $t$-phase grains have mostly transformed on the surface of the 4302 ceramic, as induced by the mild ion-cutting, revealed as the twin-structures, while it is not the case for the majority of the $t$-phase grains in the 4502 ceramic (Fig. 1). 


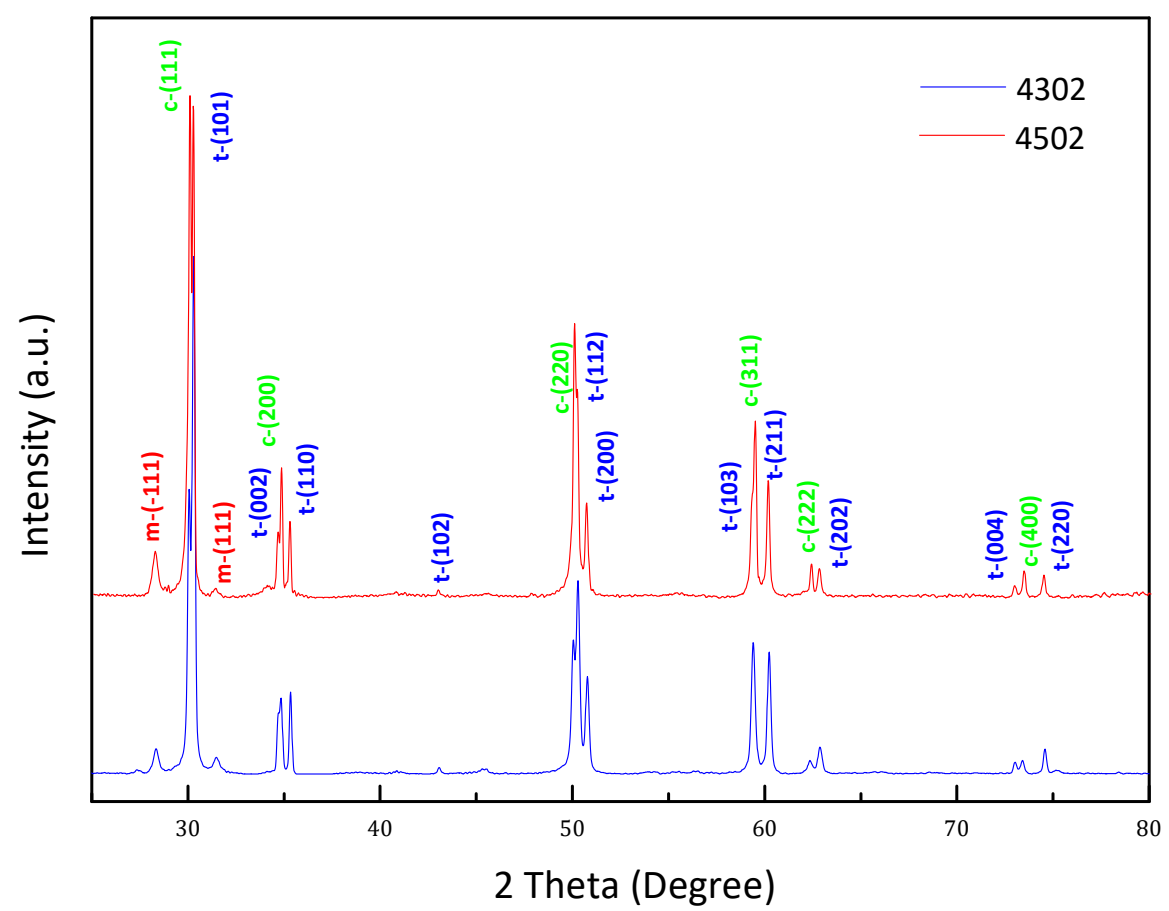

Fig. 3 XRD patterns of the 4302 and $4502 \mathrm{Nd}$ /Y-TZP ceramics obtained from the mechanical polished surface, revealing cubic (c), tetragonal ( $\mathrm{t}$ ) and monoclinic (m) $\mathrm{ZrO}_{2}$ phases.

\begin{tabular}{|c|c|c|c|c|c|c|c|}
\hline method & sample & \multicolumn{2}{|c|}{ monoclinic } & \multicolumn{2}{|c|}{ tetragonal } & cubic & \\
\hline \multirow{2}{*}{ XRD } & 4302 & \multicolumn{2}{|c|}{$12.8 \%$} & \multicolumn{2}{|c|}{$52.9 \%$} & $34.3 \%$ & - \\
\hline & 4502 & \multicolumn{2}{|c|}{$18.6 \%$} & \multicolumn{2}{|c|}{$37.7 \%$} & $43.7 \%$ & - \\
\hline & & dark & \multicolumn{2}{|c|}{ transformed } & gray & bright & hole \\
\hline \multirow{2}{*}{ SEM } & 4302 & $13.5 \%$ & \multicolumn{2}{|c|}{$54.7 \%$} & $4.1 \%$ & $26.5 \%$ & $1.2 \%$ \\
\hline & 4502 & $16.5 \%$ & \multicolumn{2}{|c|}{$7.0 \%$} & $33.5 \%$ & $41.0 \%$ & $2.0 \%$ \\
\hline
\end{tabular}

Table 1 Phase fractions of $m$-, $t$ - and $c-\mathrm{ZrO}_{2}$ phases calculated from XRD patterns (Fig. 3) in comparison with quantitative analysis of $\mathrm{ZrO}_{2}$ grain contrast in SEM (such as Fig. 1) for the 4302 and $4502 \mathrm{Nd} / \mathrm{Y}$-TZP ceramics, averaged over total imaging areas of 180 and $192 \mu \mathrm{m}^{2}$ respectively.

As summarized in Table 1 , it is more revealing for the Nd/Y-TZP ceramics to compare the phase fractions obtained from high-resolution XRD patterns with the relative areas of $\mathrm{ZrO}_{2}$ grains in different contrasts from SEM images. The fraction of bright contrast corresponds to the $c$-phase in this comparison, which is also consistent with the EBSD analysis (Fig. 2). The area of gray contrast in 4502 fits rather well to $t$ - 
phase measured by XRD, while it is only the twin-structures in 4302 to match the amount of $t$-phase detected by XRD. Such twin-structures are giving a mixed gray contrast, which are similar to the "twinned features" from the stress-induced $t \rightarrow m$ transformation in Ce- and Y-TZP ceramics [16-18]. Furthermore, the phase transformation in 4302 also uplifts the surface as shown in Fig. 1. A similar phenomenon was observed in an AFM study of Ce-TZP ceramics [19]. Therefore, the grains in both the gray contrast and twin-structures correspond to the $t-\mathrm{ZrO}_{2}$ phase, while they transformed largely into $m$-phase in 4302 but untransformed in 4502 .

Dark grains may or may not correspond to the $m$-phase grains, although their fractions match rather well with the XRD results. The contrast in SEM images is not associated only to the crystal structures of $\mathrm{ZrO}_{2}$ phases, since the differences in the grain orientation and conductivity could also contribute to this contrast, which make the distinctions between $t$ and $m$ phases more complicated. Furthermore, the stabilizer elements, especially the heavier $\mathrm{Nd}$, could also contribute to the BSE contrast, hence the stabilizer concentration in different contrast grains should be measured to better understand these phase structures.

\subsection{Co-stabilizer distribution in the dual-phase microstructures}

EDS analysis were performed in various modes to reveal the distribution of $\mathrm{Y}$ and $\mathrm{Nd}$ stabilizers within the dual-phase $\mathrm{ZrO}_{2}$ microstructures. Elemental mappings of 4302 are given in Fig. 4 together with the corresponding BSE image, taken at a beam voltage of $5 \mathrm{kV}$ for better resolution. It reveals a higher level of both co-stabilizers in solid solution in the bright $c$-phase grains and a lower level of both solutes in the dark homogeneous and gray spackled grains. The distributions of both $\mathrm{Y}$ and $\mathrm{Nd}$ are rather uniform within the bright or dark/gray grains, respectively, and even an embedded dark grain could be identified from the surrounding bright grain as marked by the arrow, which is similar to the grains marked in Fig. 2. Two levels of co-stabilizers suggest that there is a bi-modal distribution of co-stabilizers in the largely dual-phase 
microstructures of $\mathrm{Nd} / \mathrm{Y}$-TZP ceramics, which is consistent with the predominant $t$ - and $c$-phases detected by XRD. The distinction between $t$ - and $m$-phases from SEM images is less important, since the $m$-phase originates all from the $t$-phase, earlier or later. Thereafter we refer them all as $t$-phase and dark grains for simplicity.
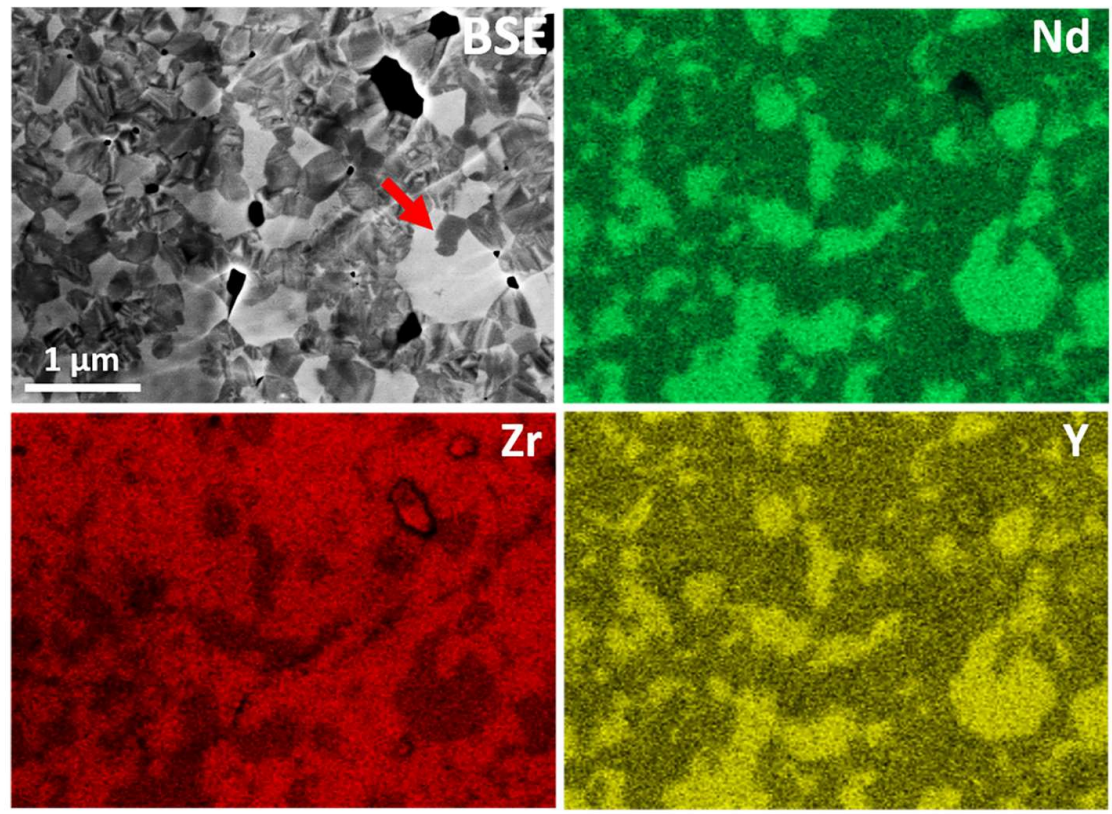

Fig. 4 Elemental EDS mapping of 4302 revealing two levels of stabilizers distributed within the dualphase microstructure of Nd/Y-TZP.

To know more about the $\mathrm{Y}$ and Nd co-stabilizer content of the bright and dark grains, their solution levels were measured by EDS analysis in individual grains. This was performed at a beam voltage of $10 \mathrm{kV}$, since the $\mathrm{Nd}$ lines could not be quantified at $5 \mathrm{kV}$ [20]. Data from about 200 grains are plotted in Fig. 5 for both ceramics, and the bright and dark grains are distinguished by open and filled symbols, respectively. The grain size was determined by measuring the area of each grain and then converting into an equivalent diameter. The correlations between the stabilizer level and the grain size are significantly different for the bright and dark grains of both ceramics. The $\mathrm{Y}$ and $\mathrm{Nd}$ content of the bright grains slowly increased over a wide range of grain sizes. Despite the same overall $\mathrm{Nd}$ content, the $\mathrm{Nd}$ content in the bright grains of 4302 is higher than that in 4502, whereas the $\mathrm{Y}$ content in these grains is substantially lower in 4302 than in 4502 to follow their doping. For the dark grains, the data are more scattered in 4302 
than in 4502, and the $\mathrm{Nd}$ content is higher than the $\mathrm{Y}$ content in one ceramics whereas it is the opposite in the other.

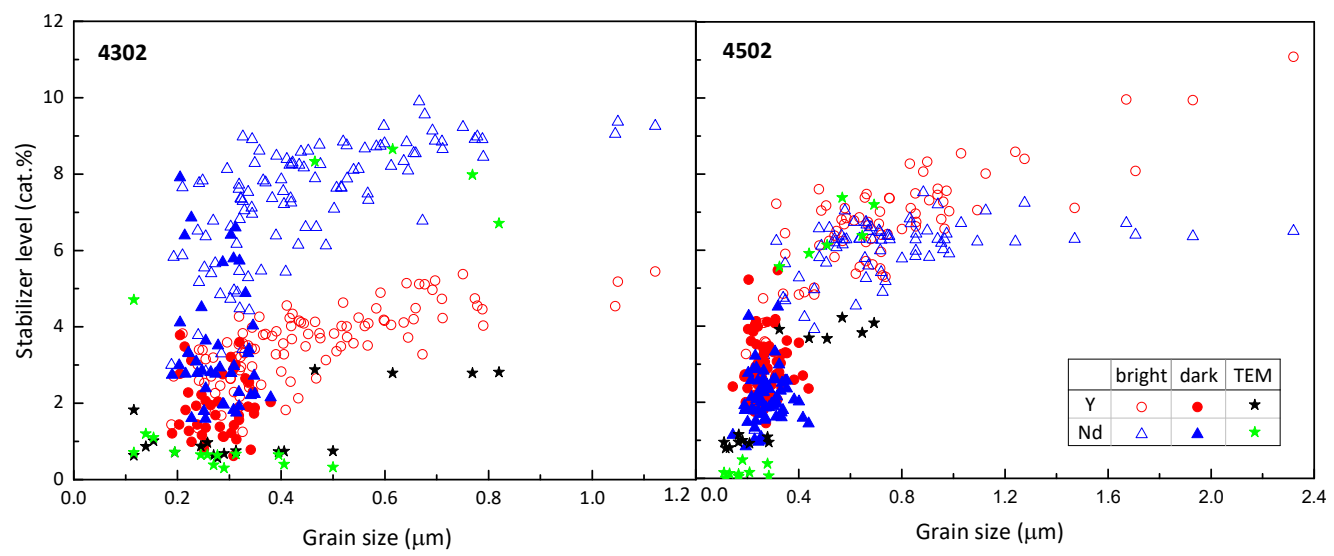

Fig. $5 \mathrm{Y}$ and $\mathrm{Nd}$ stabilizer levels in individual $\mathrm{ZrO}_{2}$ grains against the grain sizes, splitting for dark and bright grains, measured as cat.\% by EDS analysis in SEM. EDS results in TEM are also given for comparison without distinguishing the grain contrasts.

Since the effect of the electron beam penetration and broadening is more pronounced in the smaller dark grains when analyzed by SEM-EDS, EDS analysis were also done in the TEM mode, although this did not allow to differentiate the $\mathrm{ZrO}_{2}$ grains based on their contrast as in the SEM imaging. The TEM data are also plotted against the grain size in Fig. 5, which confirms and reinforced the bi-modal distribution of $\mathrm{Nd} / \mathrm{Y}$ stabilizers. The range of grain sizes analyzed in the TEM mode is much narrower and contains more small grains, which allow them to associate unmistakenly with the lower level of stabilizers of the dark grains observed in SEM. The stabilizer contents are much less scattered in the TEM analysis and significantly lower than the SEM data, especially for the dark grains, hence in clear contrast to the probe effect in the SEM analysis.

This probe effect is much less pronounced for the bright grains observed in SEM, hence the higher levels of dissolved Nd measured by TEM match rather well with those by SEM in both ceramics. The higher level of $\mathrm{Nd}$ in $c$-phase grains in the 4302 ceramic than in the 4502 ceramic, which is consistent with its higher fraction in the latter as measured by both XRD and SEM. The Y levels measured by TEM are significantly lower than those obtained by SEM, which might originate from a systematic difference in separating the overlapping $\mathrm{Y}$ and $\mathrm{Zr}$ signals in the EDS spectra by TEM and SEM, 
or in Y reference between the different EDS systems. Nevertheless, the data for the bright grains in SEM extend to a much wider grain size than the grains measured by TEM, especially for 4502. Indeed, the Y solubility increases steady with grain size at a similar rate in both ceramics, whereas the $\mathrm{Nd}$ solubility saturates relative fast against grain size. Therefore, although the bi-modal co-stabilizer distribution has been observed in both SEM and TEM, it is more reliable to use the TEM data for the lower levels of stabilizers, while it is more suitable to use the SEM data for the high levels in larger grains.

\section{Discussion}

\section{$4.1 \mathrm{ZrO}_{2}$ phase evolution by a two-stage sintering mode}

This systematic study of stabilizers in correlation with the phase relation and microstructure could bring us new insights into the sintering behavior for TZP ceramics. In a recent review on microstructure study of Y-TZP ceramics, the formation of $c$-phase was explained by a grain boundary segregation-induced phase transformation (GBSIPT) mechanism, which based on an inhomogeneous distribution of Y stabilizer within $c$ phase grains of size similar to $t$-phase grains [15]. In other words, $c$-phase was formed from $t$-phase via diffusion, after the grain growth has completed. In the present dualphase $\mathrm{ZrO}_{2}$ ceramics, the bi-modal distribution of co-stabilizers signifies rather uniform $\mathrm{Nd}$ and $\mathrm{Y}$ solution in each phase, and even more so within each grain. Such a local uniformity is hard to achieve via the grain boundary for $c$-phase with much higher levels of stabilizers. The dissolution of $\mathrm{Nd} / \mathrm{Y}$ into the $\mathrm{ZrO}_{2}$ grains of $t$ - and $c$-phases should occur under separate diffusional mode, or in separate stages of the sintering process. This is because two uniform distributions could not be realized at a same rate or time of diffusion. It is most likely that stabilizers in the $c$-phase grains were not incorporated via diffusion, but instead via grain growth. Indeed, diffusion of so high stabilizers into 
$c$-phase grains requires an equal amount of $\mathrm{Zr}$ to diffuse out of the grains, prior to the diffusion-in by the stabilizers, which is improbable at the sintering temperature. We propose an alternative two-stage sintering picture with the presence of a transient liquid-phase during sintering, which enables the large $c$-phase grains to grow directly from such liquid-phase containing both rare-earth cations in high concentrations, and highly uniform. Coating of oxide dopants onto the nanometric $m$-phase powder initiates the liquid-phase, promoted by surface structure and nanometer size of $\mathrm{ZrO}_{2}$ powder to lower the melting point by hundreds of degrees. This is effectively solutionreprecipitation process similar to $\mathrm{SiAlON}$ and $\mathrm{SiC}$ ceramics, which incorporate also additives into uniform solid-solution by liquid-phase sintering [21-23].

The transient liquid-phase can rationalize the bi-modal distribution of co-stabilizers in the dual-phase microstructures of Nd/Y-TZP in similar way. First, not only both dopants could melt to form the initial liquid, but the nanometric $m-\mathrm{ZrO}_{2}$ powder could also dissolve partially from the surface to form a liquid-phase of $\mathrm{Zr}-\mathrm{Nd}-\mathrm{Y}-\mathrm{O}$. Second, this transient liquid could enable the co-stabilizers to diffusion into $\mathrm{ZrO}_{2}$ grains, in exchange of $\mathrm{Zr}$ cations to diffuse from these grains into the liquid-phase. The exchange rate and diffusion time are both limited, permitting only low a level of stabilizers to diffuse into the grains while turning them into the $t$-phase. Third, when the ceramic body was largely densified by the transient liquid-phase and the low-level solubility of stabilizers was reached by the exchange diffusion, remaining liquid-phase carries still rather high concentration of dopants into larger pockets of the $t$-grain microstructures, from which the $c$-phase grains could precipitate directly. As demonstrated in Fig. 1, large $c$-phase grains exhibit generally concave boundaries to reveal their original nature of liquid-phase in accommodating the shapes of $t$-phase grains then already formed. The large $c$-grains develops from the liquid-phase by self-nucleation, or in the reprecipitation mode to grow onto adjacent $t$-phase grains.

In this solution-reprecipitation picture, two $\mathrm{ZrO}_{2}$ phases are developed separately in separate stages of the liquid-phase process to result in the bi-modal distribution of co-stabilizers. This two-stage sintering process is similar to the liquid-phase sintering of $\mathrm{SiAlON}$ and $\mathrm{SiC}$ ceramics, which incorporate $\mathrm{Al}_{2} \mathrm{O}_{3}$ or $\mathrm{AlN}$ dopants into the ceramic 
phase in two levels of solid-solution. This occurs not only in the liquid-phase sintered $\mathrm{SiC}$ ceramics, but also in the solid-state sintered $\mathrm{SiC}$ ceramics where the liquid-phase could only formed locally [21-23]. In the latter case, two levels of uniform AlN solution was found even in same SiC grains. This was also often observed in the present case with tiny $t$-phase grains imbedding inside $c$-phase grains, as marked by red arrows in Figs. 2, 4 and 7. Co-existence of dual-phases in such a way confirms further that the $t$ phase was formed before $c$-phase in these $\mathrm{ZrO}_{2}$ grains. The small $t$-phase grains with less stabilizers serve as seeds for the large $c$-phase grains to grow in epitaxy mode with more stabilizers.

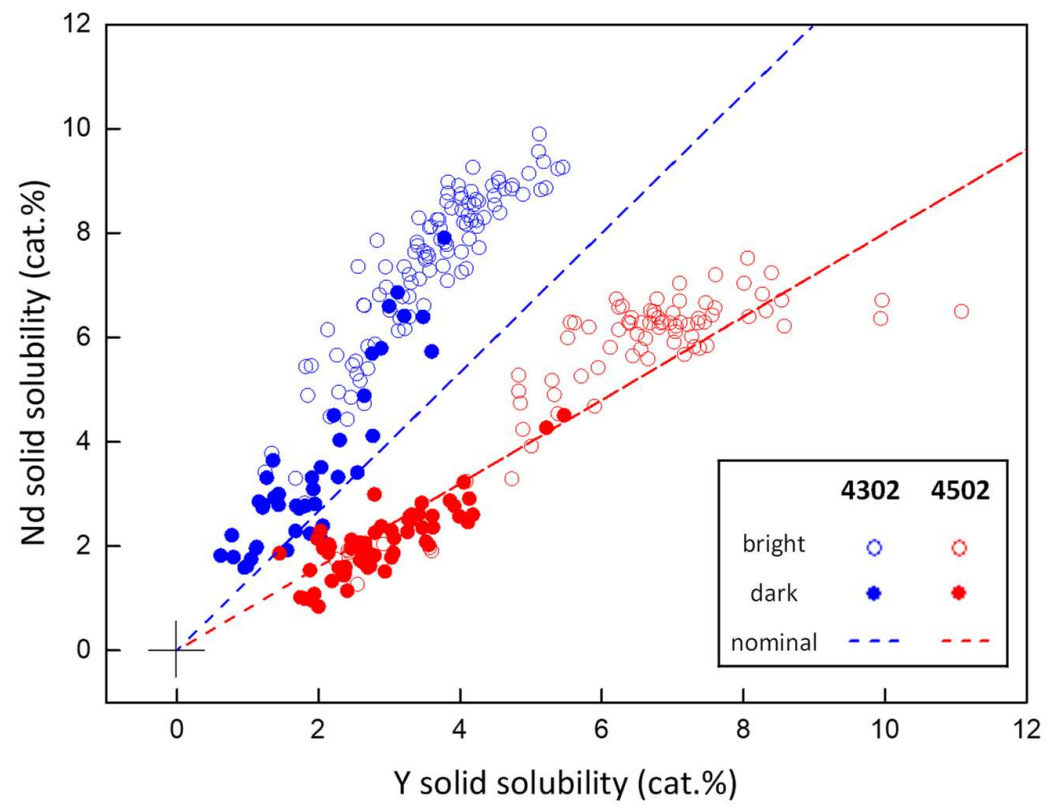

Fig. 6 Correlated distribution of co-stabilizers in the Nd-Y-TZP ceramics. Nominal ratios of the two dopants are plotted in dashed lines as references.

To reveal further information from the dual-phase microstructure developed in such two-stage sintering, we re-plotted Fig. 5 into Fig. 6 to correlate the Y and Nd stabilizer contents for every bright and dark grain in both Nd/Y-TZP ceramics. Two dashed lines represent their nominal ratios from dopants, which can serve as the references for comparison of trends between the two stabilizers. For the 4302 ceramic, stabilizer ratios between grains of both phases or contrasts fall into a common trend, while in a higher 
$\mathrm{Nd}$ :Y ratio than the nominal value. In contrast, there are two populations of stabilizers for 4502 , divided largely between the bright $c$-phase grains and dark $t$-phase grains, while the overall ratio stays with the nominal of two dopants. The bi-modal distribution of co-stabilizers and their ratio could be altered systematically by the EDS-SEM analysis when the neighboring grain is from the other phase, especially for the 4302 with relatively small grains. For 4502 with much larger bright grains, this correlation of co-stabilizers reveals a saturated trend of $\mathrm{Nd}$ in $c$-phase against $\mathrm{Y}$ level, which increases steadily over a wide range of grain size (Fig. 5). Although the transient liquidphase might start from a similar composition, the diffusion rate of both stabilizers in the $t$-phase grains should be different, which leaves a variable ratio of stabilizers in the liquid-phase hence similar trends in subsequent $c$-grain growth. Opposite to this $\mathrm{Y}$ trend in 4502, a decrease of stabilizer with grain size was reported previously in Ce-TZP, processed also by powder coating [7], which demonstrates further that the diffusional behaviors of rare-earth could correlate the phase relationship with their microstructural development during sintering process. Therefore, the re-distribution of dopants into the bi-modal distribution in the present study exposes not only the two-stage sintering process promoted by the transient liquid-phase, but also the perspective to regulate the dual-phase microstructures by optimized design of doping and sintering schedules in future.

\subsection{Constraining $t \rightarrow m$ transformation by c-phase grains}

Due to the bi-modal distribution of both stabilizers, a dual-phase microstructure dictates the mechanical behavior of $\mathrm{Nd} / \mathrm{Y}-\mathrm{TZP}$, and especially dominates the $t \rightarrow m$ phase transformation upon fracture. As revealed in a previous study of the $\mathrm{Nd} / \mathrm{Y}-\mathrm{TZP}$ system [14], sintering of 1 or $2 \mathrm{~h}$ at $1450^{\circ} \mathrm{C}$ (4301 and 4302) left no detectable amount of $m$-phase, while $m$-phase to appear after $3 \mathrm{~h}$ sintering still at $1450^{\circ} \mathrm{C}(4303)$. After storing in ambient condition for 15 years, we have detected 12.8 vol. $\% \mathrm{~m}$-phase in 4302 , and a higher fraction but still below 20 vol.\% in 4502, which should have transformed 
from the $t$-phase by the LTD effect. The original $t-\mathrm{ZrO}_{2}$ transformability reached $\sim 65$ vol. $\%$ for 4302 , and probably $55 \%$ vol. $\%$ for 4502 assuming little $m-\mathrm{ZrO}_{2}$. In contrast to rather mild LTD effect for both Nd/Y-TZP ceramics, significantly different amount of $m$-phase results from surface polishing, which shed new light into the mechanism of $t \rightarrow m$ transformation in the dual-phase microstructures. As compared in Fig. 1 and Table 1 , even the relatively mild ion-cutting for 4302 has induced a majority of twinnedfeatures from the otherwise $t$-phase grains underneath the surface, while the $t$-phase in 4502 remains largely untransformed. The extensive transformation enables some grains to lift up from the ion-cut 4302 surface to relieve the constrained strain, which is similar to the case of Ce-TZP as studied by AFM [24]. Porosity of both ceramics are also found in rather similar levels, and the most significant difference between two ceramics is the size of $c$-phase grains in the dual-phase microstructures.

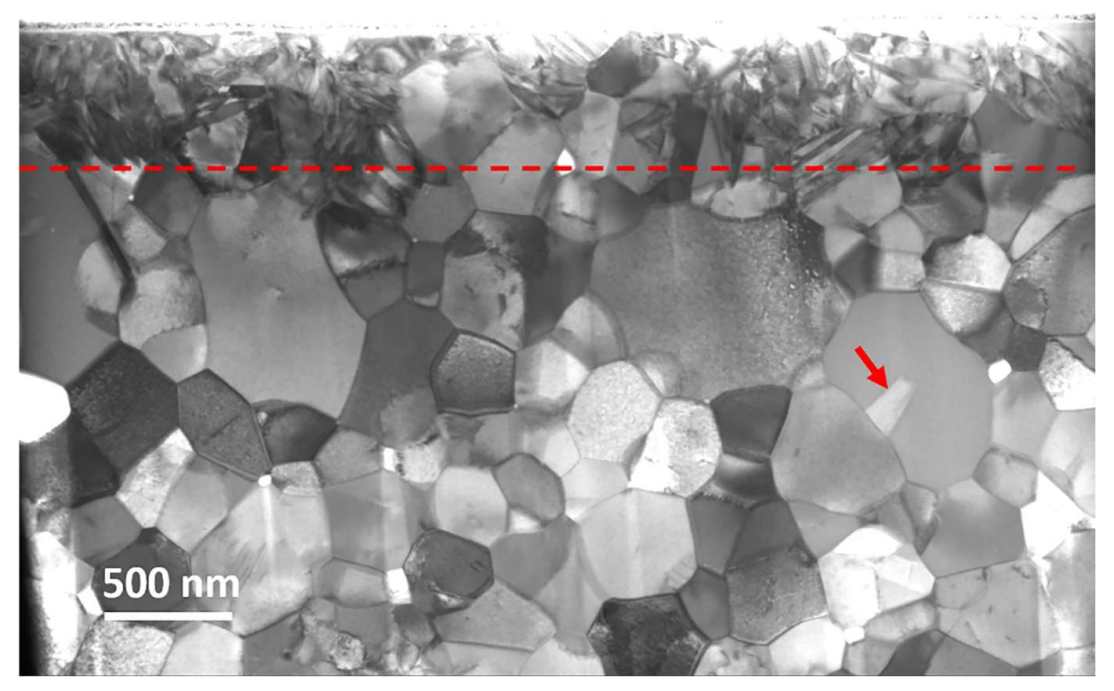

Fig. 7 Bright-field STEM image of a focused-ion-beam cross-sectioned 4302 surface demonstrating a transformed zone of twinned grains close to the surface (above the red-line) induced by mechanical polishing.

To further compare with the effect of mechanical polishing, a TEM cross-sectional specimen was prepared by focused ion beam (FIB) from the vibrational polished surface of 4302, as shown in Fig. 7. It reveals clearly that twinned grains dominate underneath the polished surface until a depth of $\sim 500 \mathrm{~nm}$, as marked by a red line. 
Below this depth, there is no sign of such twinned grain, and a small grain is again found inside a large grain as marked by the arrow, which is common to the cases marked in Fig. 2 and 4. As compared in Table 1, ion-cutting results in a 7 vol.\% $t$-phase transformation of a 4502 surface out of a total of $35 \mathrm{vol} . \%$, indicating that the transformability of most $t-\mathrm{ZrO}_{2}$ grains remains mostly constrained in 4502 under the same polishing condition as in 4302 .
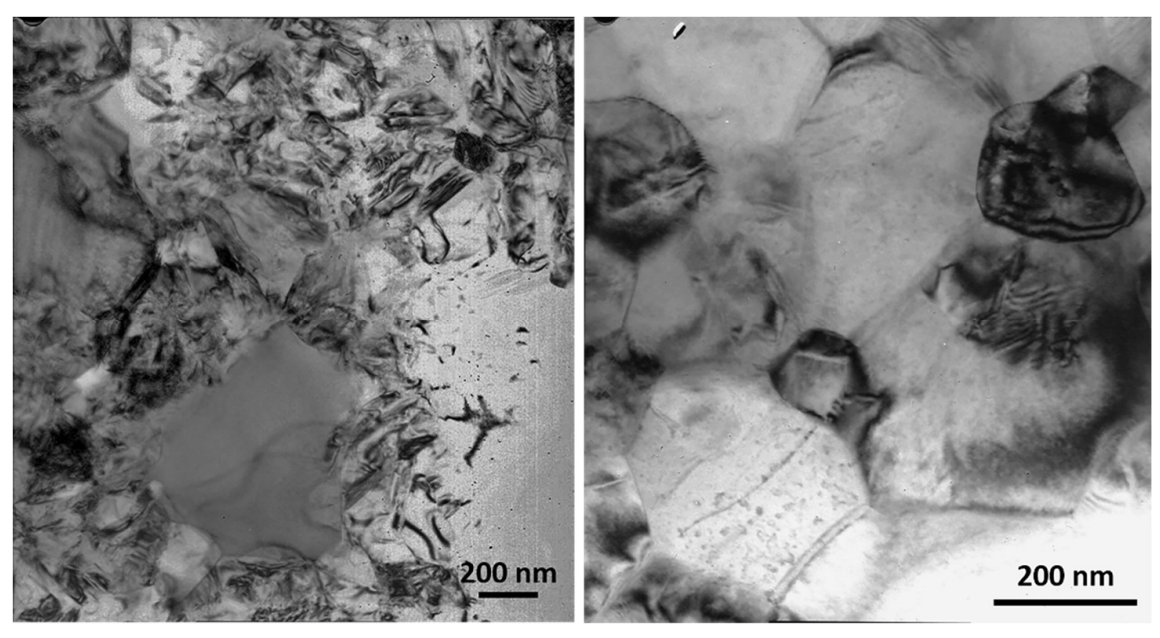

Fig. 8 Conventional bright-field TEM images of a thick (left) and thin (right) regions in Nd/Y-TZP specimens demonstrating strain fields built into small grains to constrain the transformability.

The $t$-phase grains in 4302 and 4502 are of similar size, but the grain sizes of $c$ $\mathrm{ZrO}_{2}$ are in twice the range for 4502 than 4302 , or approaching an order of difference in their volume of individual $c$-phase grains. The small $t$-phase grains with low level of $\mathrm{Y} / \mathrm{Nd}$ stabilizers are susceptible to the $t \rightarrow m$ transformation when opened up to free space. The more of small $t$-phase grains to agglomerate together, the higher for their transformability, hence it is only the $c$-phase grains in large size that could constrain effectively the transformation of neighboring $t$-phase grains. On the other hand, the stabilizer content in the $t$-phase grains of the 4302 ceramic is also lower than that in the 4502 ceramic, boosting up further their transformability. This effect will compete with the constrain effect from the $c$-phase grains on the transformation to further widen the gap of observed transformability between the two TZP ceramics. Upon cooling the $c$ - 
$\mathrm{ZrO}_{2}$ phase shrinks usually slower than the $t-\mathrm{ZrO}_{2}$ phase, hence a $c$-phase grain should generate a compressive stress into the matrix of $t$-phase to constrain the transformability in its neighborhood. In the standard TEM specimens thinned down by mild ion-milling, transformation was not found in both $\mathrm{Nd} / \mathrm{Y}$-TZP ceramics as predominant as in FIB sample, as demonstrated in Fig. 8. Instead, strain contrasts are often observed to develop into the small grains especially surrounding a large grain free from such strain (left panel of Fig. 8). The small grains could accommodate the compressional stress by elastic deformation within the thin TEM foils, which is in contrast to the SEM observation opening up to free space especially in 4302. It is the combination of differences in Young's modulus and in grain size between the two phases to dictate the transformability of the $t$-phase grains in the neighborhood.

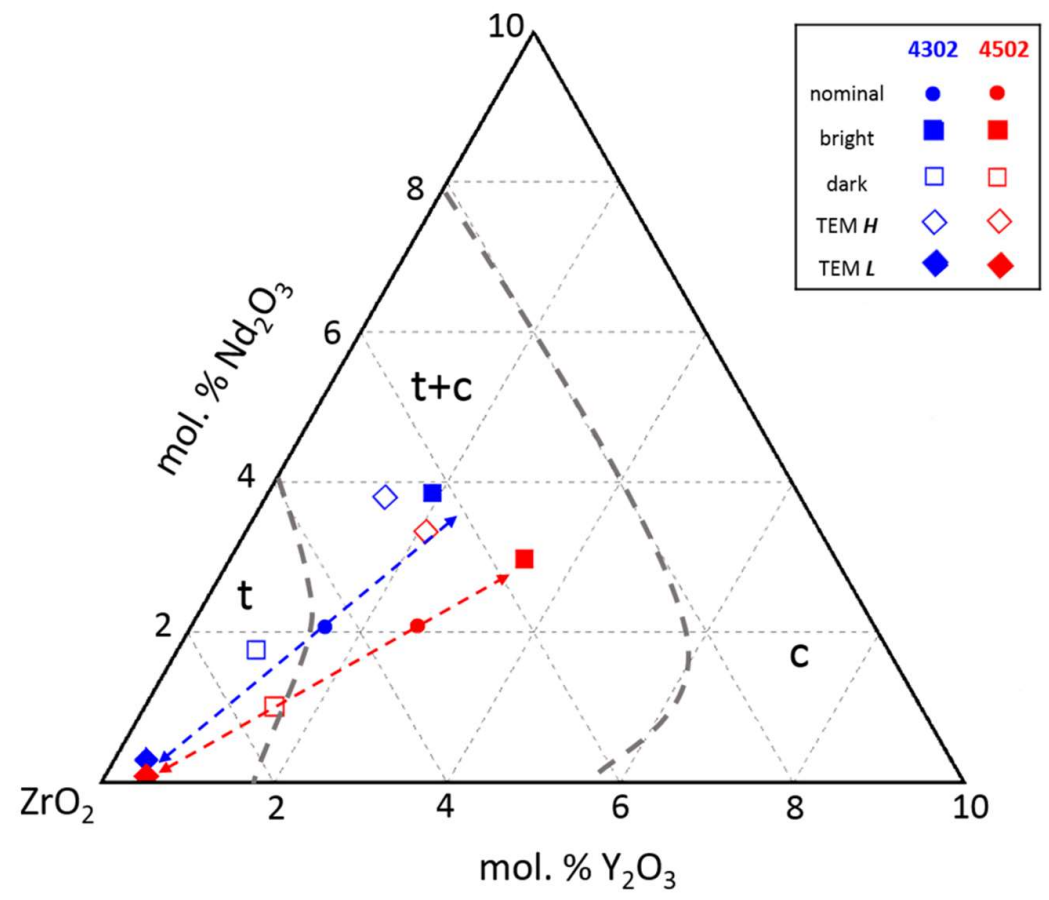

Fig. 9 The two-phase relationship of both $\mathrm{Nd} / \mathrm{Y}$-TZP ceramics plotted in the ternary $\mathrm{ZrO}_{2}-\mathrm{Nd}_{2} \mathrm{O}_{3}-$ $\mathrm{Y}_{2} \mathrm{O}_{3}$ phase diagram, taken averages from EDS results in SEM and TEM modes (see Fig. 5 and Table $\mathrm{S} 1)$. Data are more reliable for the $t-\mathrm{ZrO}_{2}$ phase from the low level in TEM, and for $c-\mathrm{ZrO}_{2}$ phases from bright grains in SEM, respectively; they are represent by filled symbols. The nominal stabilizer compositions are marked by round dots, from which the re-distribution of co-stabilizers separate the $\mathrm{ZrO}_{2}$ structures into two phases. Two phase boundaries are calculated for $1450^{\circ} \mathrm{C}$ in Ref. [12]. 
After revealing the bi-modal distribution of co-stabilizers, we can renew the dualphase relationship into the ternary $\mathrm{ZrO}_{2}-\mathrm{Nd}_{2} \mathrm{O}_{3}-\mathrm{Y}_{2} \mathrm{O}_{3}$ phase diagram, as shown for the $\mathrm{ZrO}_{2}$-rich corner in Fig. 9. The nominal composition of 4302 lies almost on the first phase boundary between the $t$-and mixed-phase zones, and 4502 locates rather deep into the predicted dual-phase zone. Although we plotted both sets of stabilizer contents measured by SEM-EDS and TEM-EDS as all averaged in Table S1, the SEM data from bright grains are more representative for $c-\mathrm{ZrO}_{2}$ phase, and the low level TEM data (TEM L) for $t-\mathrm{ZrO}_{2}$ phase, as compared in the results section. The nominal compositions in two TZP ceramics from starting powder are both on the tie-lines of two $\mathrm{ZrO}_{2}$ phases, demonstrating that a clear "phase separation" has occurred for the dualphase relationship. This phase-separation starts from very close compositions for $t$ phase to diverge towards rather different composition for $c$-phase, manifesting a dictating effect of the transient liquid-phase to guide the stabilizer distribution and the dual-phase relation in the two-stage process. It is the powder coating that enables the transient liquid-phase to form early and in uniform composition, which enables the first stage to start early to leave ample time for diffusion into uniform levels, both in the tphase and in the transient liquid-phase to reach a clear correlation between the costabilizers and the dual-phase microstructures. Other processing routes may not homogenize the stabilizers in similar dual-microstructures into clear-cut two-stages. Whether the stabilizer level is sufficient to stabilize or over-stabilize the $t$-phase depends also and more on the $c$-phase developed in the second-stage. Further elaboration of co-stabilizer distributions and phase relations in future will lead to a systematic understanding and optimization of the structure-property relations for TZP ceramics.

\section{Conclusions}

By quantitative analysis of stabilizer distribution within two Nd/Y-TZP ceramics, 
we reveal a bi-modal distribution within the dual-phase microstructures. In comparison of various SEM observation and XRD analysis, different behaviors of $t \rightarrow m$ transformation on polished surface are found to originate from a difference in grain size distribution between the two ceramics of similar dual-phase relations. A transient liquid-phase was proposed to rationalize the formation of $t$ - and $c-\mathrm{ZrO}_{2}$ phases in a twostage sintering process, which explains the re-distribution of stabilizers into the bimodal distribution to develop the common dual-phase microstructures. This study further demonstrates that the transformability of $t$-phase grains could be constrained from the neighboring $c$-phase grains especially in much large size. Such inherent association of the dual-phase relationship and their microstructures opens new perspectives to tailor the dual-phase microstructures of TZP ceramics for better performance.

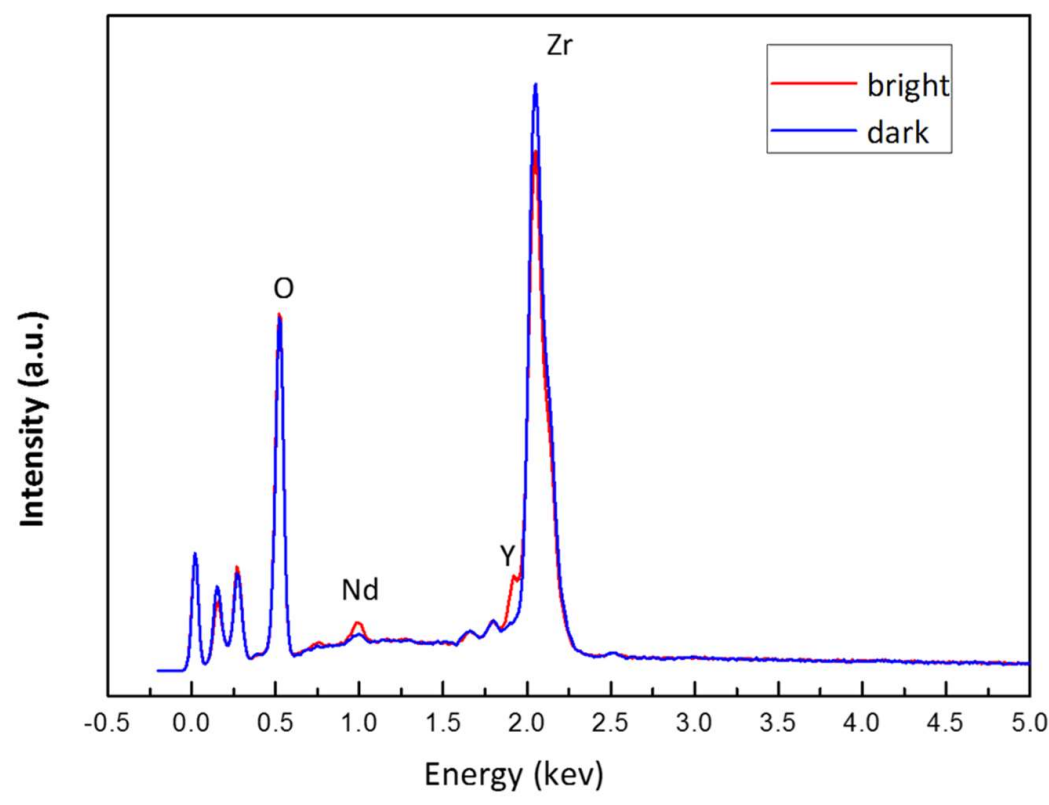

Fig. S1 Typical EDS spectra of bright and dark grains in 4502, data presented in Fig. 5 are processed from similar spectra. 


\begin{tabular}{|c|c|c|c|c|c|}
\hline \multirow{2}{*}{ Samples } & stabilizers & bright & dark & TEM $\boldsymbol{H}$ & TEM $\boldsymbol{L}$ \\
\hline \multirow{2}{*}{4302} & $\mathrm{Y}_{2} \mathrm{O}_{3}$ & $1.88 \pm 0.5$ & $0.99 \pm 0.4$ & $1.38 \pm 0.2$ & $0.37 \pm 0.1$ \\
\cline { 2 - 6 } & $\mathrm{Nd}_{2} \mathrm{O}_{3}$ & $3.90 \pm 0.9$ & $1.80 \pm 0.8$ & $3.83 \pm 0.8$ & $0.31 \pm 0.1$ \\
\hline \multirow{2}{*}{4502} & $\mathrm{Y}_{2} \mathrm{O}_{3}$ & $3.45 \pm 0.9$ & $1.49 \pm 0.4$ & $2.07 \pm 0.1$ & $0.48 \pm 0.1$ \\
\cline { 2 - 6 } & $\mathrm{Nd}_{2} \mathrm{O}_{3}$ & $3.02 \pm 0.8$ & $1.08 \pm 0.4$ & $3.39 \pm 0.4$ & $0.09 \pm 0.07$ \\
\hline
\end{tabular}

Table S1 High and low levels of stabilizers in the two-phase Nd/Y-TZP ceramics, measured by EDS analysis in TEM or averaged from bright/dark grains in SEM. They are all plotted in Fig. 9 for comparison and further discussion.

\section{Acknowledgements}

This work is financially supported by the National Natural Science Foundation of China (Grant No. 51532006), Science and Technology Commission of Shanghai Municipality (16DZ2260601) and the 111 Project (D16002). The authors would like to thank Dr. Tao Xu and Prof. Pei-Ling Wang for helpful discussion. We also thank Mr. Hai-Feng Gao and Dr. Xin-Xin Yang for their help in FIB and XRD experiments.

\section{References}

[1] J. Chevalier, L. Gremillard, A.V. Virkar, D.R. Clarke, The Tetragonal-Monoclinic Transformation in 
Zirconia: Lessons Learned and Future Trends, Journal of the American Ceramic Society 92(9) (2009) 1901-1920.

[2] B. Cales, Zirconia as a sliding material: histologic, laboratory, and clinical data, Clinical Orthopaedics \& Related Research 379(379) (2000) 94-112.

[3] F.F. Lange, Transformation-toughened $\mathrm{ZrO}_{2}$ : correlations between grain size control and composition in the system $\mathrm{ZrO}_{2}-\mathrm{Y}_{2} \mathrm{O}_{3}$, Journal of the American Ceramic Society 69(3) (1986) 240-242.

[4] L. Ruiz, M.J. Readey, Effect of heat-treatment on grain size, phase assemblage, and mechanical properties of 3 mol\% Y-TZP, Journal Of the American Ceramic Society 79(9) (1996) 2331-2340.

[5] A. Suresh, M.J. Mayo, W.D. Porter, C.J. Rawn, Crystallite and grain-size-dependent phase transformations in yttria-doped zirconia, Journal of the American Ceramic Society 86(2) (2003) 360-362. [6] B. Basu, Toughening of yttria-stabilised tetragonal zirconia ceramics, International Materials Reviews 50(4) (2005) 239-256.

[7] P.A. Fang, H. Gu, P.L. Wang, Effect of powder coating on stabilizer distribution in $\mathrm{CeO}_{2}$-stabilized $\mathrm{ZrO}_{2}$ ceramics, Journal Of the American Ceramic Society 88(7) (2005) 1929-1934.

[8] F. Zhang, M. Inokoshi, M. Batuk, J. Hadermann, I. Naert, M.B. Van, J. Vleugels, Strength, toughness and aging stability of highly-translucent Y-TZP ceramics for dental restorations, Dental Materials Official Publication of the Academy of Dental Materials 32(12) (2016).

[9] F. Zhang, M. Inokoshi, M. Batuk, J. Hadermann, I. Naert, M.B. Van, J. Vleugels, Highly-translucent, strong and aging-resistant 3Y-TZP ceramics for dental restoration by grain boundary segregation, Acta Biomaterialia 16(1) (2015) 215-222.

[10] M.M.R. Boutz, A.J.A. Winnubst, B.V. Langerak, R.J.M.O. Scholtenhuis, K. Kreuwel, A.J. Burggraaf, The effect of ceria co-doping on chemical stability and fracture toughness of Y-TZP, Journal of Materials Science 30(7) (1995) 1854-1862.

[11] S.G. Huang, J. Vleugels, L. Li, O. Van der Biest, P.L. Wang, Composition design and mechanical properties of mixed $(\mathrm{Ce}, \mathrm{Y})$-TZP ceramics obtained from coated starting powders, Journal of the European Ceramic Society 25(13) (2005) 3109-3115.

[12] J. Vleugels, T. Xu, S.G. Huang, Y.M. Kan, P.L. Wang, L. Li, O. Van der Biest, Characterization of (Nd,Y)TZP ceramics prepared by a colloidal suspension coating technique, Journal Of the European Ceramic Society 27(2-3) (2007) 1339-1343.

[13] T. Xu, J. Vleugels, O. Van der Biest, Y.M. Kan, P.L. Wang, Phase stability and mechanical properties of TZP with a low mixed $\mathrm{Nd}_{2} \mathrm{O}_{3} / \mathrm{Y}_{2} \mathrm{O}_{3}$ stabiliser content, Journal Of the European Ceramic Society 26(7) (2006) 1205-1211.

[14] T. Xu, J. Vleugels, O. Van der Biest, P.L. Wang, Mechanical properties of $\mathrm{Nd}_{2} \mathrm{O}_{3} / \mathrm{Y}_{2} \mathrm{O}_{3}$-coated zirconia ceramics, Mater. Sci. Eng. A-Struct. Mater. Prop. Microstruct. Process. 374(1-2) (2004) 239-243.

[15] H. Yoshida, Y. Ikuhara, Review: microstructure-development mechanism during sintering in polycrystalline zirconia AU - Matsui, Koji, International Materials Reviews 63(6) (2018) 375-406.

[16] T. Xu, P.L. Wang, P.A. Fang, Y.M. Kan, L.D. Chen, J. Vleugels, O. Van der Biest, J. Van Landuyt, Phase assembly and microstructure of $\mathrm{CeO}_{2}$-doped $\mathrm{ZrO}_{2}$ ceramics prepared by spark plasma sintering, Journal Of the European Ceramic Society 25(15) (2005) 3437-3442.

[17] G.R. Hugo, B.C. Muddle, R.H.J. Hannink, The Tetragonal to Monoclinic Transformation in CeriaZirconia, Materials Science Forum, 1990, pp. 357-362.

[18] B.C. Muddle, R.H.J. Hannink, Crystallography of the Tetragonal to Monoclinic Transformation in MgO - Partially - Stabilized Zirconia, Journal of the American Ceramic Society 69(7) (2010) 547-555.

[19] S. Deville, H. El Attaoui, J. Chevalier, Atomic force microscopy of transformation toughening in ceria- 
stabilized zirconia, Journal of the European Ceramic Society 25(13) (2005) 3089-3096.

[20] W. Chen, D.L. Hu, H. Gu, J.J. Xing, Systematic Characterization of Microstructure and Phase evolution in Co-doped Zirconia Ceramics by Scanning Electron Microscopy, Journal of the Chinese Ceramic Society (2019) xxxxx..

[21] R. Huang, H. Gu, J.X. Zhang, D.L. Jiang, Effect Of $\mathrm{Y}_{2} \mathrm{O}_{3}-\mathrm{Al}_{2} \mathrm{O}_{3}$ ratio on inter-granular phases and films in tape-casting alpha-SiC with high toughness, Acta Materialia 53(8) (2005) 2521-2529.

[22] J.F. Hu, H. Gu, Z.M. Chen, S. Tan, D. Jiang, M. Rühle, Core-shell structure from the solutionreprecipitation process in hot-pressed AIN-doped SiC ceramics, Acta Materialia 55(16) (2007) 5666-5673. [23] R. Huang, H. Gu, F. Aldinger, Intergranular Oxynitride to Regulate Solution-Reprecipitation Process in Gas-Pressure-Sintered SiC Ceramics with AIN- $\mathrm{Y}_{2} \mathrm{O}_{3}$ Additives, Advanced Engineering Materials (2018) xxxxx.

[24] S. Deville, G. Guénin, J. Chevalier, Martensitic transformation in zirconia: Part I. Nanometer scale prediction and measurement of transformation induced relief, Acta Materialia 52(19) (2004) 5697-5707. 